\title{
Loss of interleukin17RA expression is associated with tumour progression in colorectal carcinoma
}

\begin{abstract}
Interleukin-17 (IL-17) is a pro-inflammatory cytokine found in various cancers. Current evidence indicates that IL-17 plays a vital role in tumour initiation and progression in colorectal carcinoma (CRC) via binding with its receptor, IL-17RA. However, the association between clinicopathological features and presence of IL-17 and IL-17RA protein in primary CRC tissues remains unclear. This study also investigates the difference between the presence of IL17 and IL-17RA in the paired tumour tissues versus adjacent normal tissues. The presence of IL-17RA and IL-17 protein in primary CRC tissues was determined by immunohistochemistry. Associations between clinicopathological features and IL-17RA and IL-17 immunoreactivity, were analyzed by $\chi 2$ tests. We found that both IL-17RA $(p=0.001)$ and IL-17 $(p=0.025)$ in tumour cells of primary CRC tissues was significantly lower as compared to adjacent normal tissue. Positive immunoreactivity for IL-17RA and IL-17 were detected in $51.0 \%$ and $16.8 \%$ of tumour tissues, respectively. Furthermore, negative immunoreactivity of IL-17R was significantly associated with advanced stage according to TNM classifier $(\mathrm{p}=0.027)$, high grade of tumour $(p=0.019)$, increased depth of tumour invasion $(p=0.023)$ and vascular invasion $(p=0.039)$. Positive IL-17 immunoreactivity was associated with advanced stage $(p=0.008)$ and lymph node metastasis $(\mathrm{p}=0.008)$. Thus, this study suggests that the loss of IL-17RA expression occurs as tumour progresses and this may predict the aggressiveness of tumour whilst expression of IL-17 promotes tumour progression and lymph node metastasis. Thus, loss of IL17RA could be a useful prognostic biomarker for tumour progression in CRC patients.
\end{abstract}

Keyword: IL-17; IL-17RA; Colorectal carcinoma; Prognostic biomarker; Tumour progression 